\title{
Outcome of ventilatory support for acute respiratory failure in motor neurone disease
}

\author{
M D Bradley, R W Orrell, J Clarke, A C Davidson, A J Williams, D M Kullmann, \\ N Hirsch, R S Howard
}

See end of article for authors' affiliations

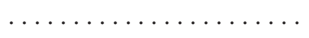

Correspondence to: Dr Robin S Howard, Batten-Harris Unit, National Hospital for Neurology and Neurosurgery, Queen

Square, London WCIN 3BG, UK:

r.howard@ion.ucl.uk.ac

Received 26 September 2001

In revised form

24 January 2002

Accepted

13 February 2002

\begin{abstract}
Objectives: To review the outcome of acute ventilatory support in patients presenting acutely with respiratory failure, either with an established diagnosis of motor neurone disease (MND) or with a clinical event where the diagnosis of MND has not yet been established.

Methods: Outcome was reviewed in 24 patients with respiratory failure due to MND who received endotracheal intubation and intermittent positive pressure ventilation either at presentation or as a result of the unexpected development of respiratory failure. Patients presenting to local hospitals with acute respiratory insufficiency and requiring tracheal intubation, ventilatory support, and admission to an intensive therapy unit (ITU) before transfer to a regional respiratory care unit were selected. Clinical features of presentation, management, and outcome were studied.

Results: 24 patients with MND were identified, all being intubated and ventilated acutely within hours of presentation. 17 patients $(71 \%)$ were admitted in respiratory failure before the diagnosis of MND had been made; the remaining seven patients $(29 \%)$ were already known to have MND but deteriorated rapidly such that intubation and ventilation were initiated acutely. Seven patients $(29 \%)$ died on ITU (between seven and 54 days after admission). 17 patients (71\%) were discharged from ITU. 16 patients $(67 \%)$ received long term respiratory support and one patient required no respiratory support following tracheal extubation. The daily duration of support that was required increased gradually with time.

Conclusion: When a patient with MND is ventilated acutely, with or without an established diagnosis, independence from the ventilator is rarely achieved. Almost all of these patients need long term ventilatory support and the degree of respiratory support increases with time as the disease progresses. The aim of management should be weaning the patient to the minimum support compatible with symptomatic relief and comfort. Respiratory failure should be anticipated in patients with MND when the diagnosis has been established.
\end{abstract}

$\mathrm{T}$ he onset of motor neurone disease (MND) is usually insidious with a diverse range of clinical phenotypes, but the disease has a poor prognosis with death ensuing in the majority of cases between two and four years from diagnosis. ${ }^{1}$ The most common cause of death is respiratory failure ${ }^{2-4}$ due to diaphragmatic, ${ }^{5-7}$ intercostal, and accessory muscle weakness. In addition poor bulbar control and cough cause an increased risk of pulmonary aspiration. ${ }^{8}$

It has been increasingly recognised that the provision of respiratory support can provide symptomatic relief and increase life expectancy. ${ }^{9-14}$ However, these benefits must be balanced against the difficulties of compliance in severely disabled patients, ${ }^{13}{ }^{15}$ the demands on carers and relatives, ${ }^{16}$ the practical problems of administrating ventilatory support, the risk of iatrogenic problems, increasing dependence on ventilatory support ${ }^{11}$ causing distressing and unwanted prolongation of life, and difficulties in managing the terminal stages in these patients. While many of these difficulties can be avoided by careful discussion with patients and their carers, ${ }^{16}{ }^{17}$ many patients delay or wish to avoid taking decisions about embarking on ventilatory support. Elective ventilatory support is usually administered non-invasively through masks (facial or nasal), mouthpieces, or nasal pillows, and initially it is usually used for improving respiratory function during sleep. Non-invasive administration has advantages because it allows speech and oral feeding, has lower costs, and leads to fewer respiratory infections, ${ }^{18}$ but it may not be desirable in patients with severe bulbar weakness, in those with facial abnormalities, or where aspiration has already occurred ${ }^{13}$. Non-invasive ventilatory support may improve survival in $\mathrm{MND},{ }^{19} 20$ reduce the work of breathing, promote good gas exchange, and improve quality of sleep $^{21}$; this often results in improvement of daytime symptoms such as breathlessness and excessive sleepiness. Some patients, however, may require ventilatory support for increasing periods while awake or even continuously and may choose to undergo tracheostomy. ${ }^{14}$ This facilitates ventilatory support by reducing respiratory dead space and does not affect speech or swallowing provided an uncuffed tracheostomy tube is used. However, an uncuffed tube does not afford protection against the risk of pulmonary aspiration.

Although acute respiratory insufficiency is uncommon, patients may present with it. ${ }^{1022-24}$ They may be distressed, dyspnoeic, confused, and drowsy. Arterial blood gas analysis shows the hypoxaemia and hypercarbia of type II respiratory failure. Frequently, such patients are in extremis and are rapidly intubated with a tracheal tube and receive positive pressure ventilation (PPV). Often MND in patients who present in this fashion is undiagnosed and the diagnosis may not become apparent following subsequent clinical examination until the patient has been admitted to the intensive therapy unit (ITU) and received a full clinical assessment. Even in those patients known to have MND, the admitting

Abbreviations: IPPV intermittent positive pressure ventilation; ITU, intensive therapy unit; MND, motor neurone disease; NPD, not previously diagnosed; PD, previously diagnosed; PPV, positive pressure ventilation 


\begin{tabular}{|c|c|c|}
\hline & $\begin{array}{l}\text { Previously } \\
\text { diagnosed }(n=7)\end{array}$ & $\begin{array}{l}\text { Not previously } \\
\text { diagnosed }(n=17)\end{array}$ \\
\hline Sex male:female & $5: 2$ & $15: 2$ \\
\hline Mean (SD) age at first symptoms (years) & $57.8(7.3)$ & $67.1(6.1)$ \\
\hline Mean (SD) age at intubation (years) & $60.3(7.1)$ & $67.8(6.2)$ \\
\hline Time (range) between onset of symptoms and intubation (months) & $24(6-84)$ & $6(0-30)$ \\
\hline \multicolumn{3}{|l|}{ Site of onset } \\
\hline Lower limb & 5 & 4 \\
\hline Upper limb & 1 & 3 \\
\hline Upper and lower limbs & & 3 \\
\hline Bulbar & 1 & 4 \\
\hline Diaphragm & & 3 \\
\hline \multicolumn{3}{|l|}{ Initial diagnosis } \\
\hline ALS & 6 & 10 \\
\hline $\mathrm{PBP} *$ & 1 & 4 \\
\hline $\mathrm{PMA}^{*}$ & & 3 \\
\hline \multicolumn{3}{|l|}{ Cause of deterioration } \\
\hline Pneumonia & 5 & 4 \\
\hline Rapidly progressive dyspnoea & 1 & 6 \\
\hline Under investigation when developed respiratory arrest & & 5 \\
\hline Nocturnal seizure & & 1 \\
\hline After routine general anaesthetic & & 1 \\
\hline Intubated overseas & 1 & \\
\hline
\end{tabular}

doctors may perform tracheal intubation and initiate ventilation either because the patient has expressed a wish for full support in the event of respiratory failure or because the patient or relatives have expressed no preference and the admitting doctors are uncertain about their wishes or the patient's prognosis.

In the present study we have reviewed the outcome of 24 patients with respiratory failure due to MND who underwent tracheal intubation and intermittent PPV (IPPV) as an emergency procedure either at presentation or as a result of the sudden unexpected development of respiratory insufficiency.

\section{METHODS}

We retrospectively reviewed all the case notes of patients with MND admitted to the Batten-Harris Unit at the National Hospital for Neurology and Neurosurgery, London, and the LaneFox Unit at St Thomas' Hospital, London, United Kingdom, between 1980 and 2000. Those patients presenting to local hospitals with acute respiratory insufficiency and requiring tracheal intubation, ventilatory support, and admission to an ITU before transfer were analysed. The diagnosis of MND was always made or confirmed by a consultant neurologist using standard clinical assessment, electromyography, and sometimes further investigations to exclude other diagnoses. ${ }^{25}$ Patients were followed up until December 2000 or death, whichever occurred sooner.

Clinical information extracted from all the medical records included the onset, duration, and nature of all symptoms; whether the diagnosis of MND was known before the initiation of artificial ventilation; the cause of respiratory decline; the course and duration of the time spent on ITU; and the mode of respiratory support. The quantity and quality of information available for each patient varied depending on individual circumstances, such as concurrent illness and social background.

Statistical analysis was performed using $t$ test (two tailed). Results are expressed as mean (SD).

\section{RESULTS}

\section{Presentation}

Twenty four patients with MND were identified, of whom 20 $(83 \%)$ were male. All had undergone tracheal intubation and were ventilated acutely within hours of presentation. Seventeen patients $(71 \%)$ were admitted in respiratory failure before the diagnosis of MND had been made (not previously diagnosed (NPD)); the remaining seven patients were already known to have MND (previously diagnosed (PD)) but deteriorated rapidly such that tracheal intubation and ventilation were initiated acutely (table 1 ).

The mean age at onset of symptoms (including undiagnosed weakness or weight loss) was 64 years (range 50-79). Age at onset of symptoms of muscle weakness was significantly lower $(\mathrm{p}<0.01)$ in the PD $(57.8(7.3)$ years, $\mathrm{n}=7$ ) than in the NPD group (67.1 (6.1) years, $\mathrm{n}=17$ ). One patient had no discernible history of symptoms before the onset of respiratory failure. All the patients in the NPD group and five of the seven patients in the PD group were mobile before the development of ventilatory failure.

Symptoms in six of the seven patients in the PD group had begun in their limbs and one patient had a predominantly bulbar onset. Patients in the NPD group had a more mixed symptomatology: 10 patients had disease onset in the limbs and four bulbar onset, two patients had complained of dyspnoea, and one patient presented in respiratory failure with no preceding history. Nineteen patients had amyotrophic lateral sclerosis and five had progressive bulbar palsy but had progressively developed limb signs.

The mean age at onset of acute respiratory failure for all patients was 66 years (range 51-80). The age at onset of respiratory failure was significantly lower $(p=0.03)$ in the PD group (mean $60.3(7.1)$ years, $\mathrm{n}=7$ ) than in the NPD group (mean $67.8(6.2)$ years, $\mathrm{n}=17$ ).

Table 1 summarises the causes of the acute deterioration and presentation in respiratory failure. Of the seven patients in the PD group, five were suffering from pneumonia on clinical and radiographic grounds, one had progressive dyspnoea associated with rapid disease progression, and one patient had undergone tracheal intubation overseas before transfer back to the United Kingdom (the precise circumstances were unclear). Of the 17 patients in the NPD group, four had pneumonia, six had rapidly progressive dyspnoea, and five were inpatients under investigation for other symptoms (such as weight loss or weakness) when they had a respiratory arrest. In one of these cases this followed a nocturnal seizure and 
Table 2 Outcomes of 24 patients with MND at the end of the study period

\begin{tabular}{llll}
\hline & $\begin{array}{l}\text { Previously } \\
\text { diagnosed }(n=7)\end{array}$ & $\begin{array}{l}\text { Not previously } \\
\text { diagnosed }(n=17)\end{array}$ & Total $(\mathbf{n = 2 4 )}$ \\
\hline Died on ITU while receiving ventilatory support & $2(29 \%)$ & $5(29 \%)$ & $7(29 \%)$ \\
Time (range) spent on ITU before death (days) & $25-56$ & $7-54$ & $7-56$ \\
Discharged from ITU with respiratory support & $5(71 \%)$ & $12(71 \%)$ & $17(71 \%)$ \\
Fully extubated and discharged with no support & 0 & $1(6 \%)$ & $1(4 \%)$ \\
IPPV through tracheostomy & $4(57 \%)$ & $9(53 \%)$ & $13(54 \%)$ \\
IPPV through facemask & $1(14 \%)$ & $1(6 \%)$ & $2(8 \%)$ \\
Rocking bed & 0 & $1(6 \%)$ & $1(4 \%)$ \\
\hline \multirow{2}{*}{ IPPV, intermittent positive pressure ventilation; ITU, intensive therapy unit } & \\
\hline
\end{tabular}

another patient could not be extubated after a routine general anaesthetic.

The mean time between symptom onset and respiratory failure was six months (range $0-30, n=17$ ) for the NPD group and 24 months for the PD group (range 6-84, $\mathrm{n}=7$ ). In the PD group the mean time between onset of first symptoms and diagnosis was 12 months (range 5-24), and the mean duration between diagnosis and respiratory failure was 12 months (range 2-60). There was no significant difference in the age of onset of respiratory failure between men and women. Tracheostomy was performed in all 24 patients; this was undertaken in the referring hospital in eight and in the specialist centre in 16 .

\section{Progress}

Table 2 shows the final outcome for all 24 patients. Six patients $(29 \%)$ died on ITU and one following transfer to a longer stay unit. In each case the patient died following the development of a severe bronchopneumonia and after the patient and carer had agreed antibiotics should not be administered. These patients died between 7 and 54 days following admission to ITU. Seventeen patients $(71 \%)$ were discharged from ITU. Sixteen patients $(67 \%)$ received long term respiratory support and one $(4 \%)$ patient required no respiratory support following tracheal extubation. Thirteen patients (54\%) received IPPV through a tracheostomy and two through a nasal mask. One patient used a rocking bed at night. The daily duration of ventilatory support required varied from 8 hours overnight to 24 hours continuously. The period of support gradually increased with time.

\section{PD group of patients}

All seven of the patients in the PD group were established on IPPV through an uncuffed tracheostomy (table 2). Two patients died on ITU after two months. These patients had severe limb and respiratory muscle weakness and it was not possible to wean them from mechanical ventilation. They both developed aspiration pneumonia and, after discussion with the patient, antibiotics were not administered. The terminal stages were managed in close liaison with palliative care colleagues in both centres. Five patients $(71 \%)$ were discharged from ITU requiring IPPV (one to a local hospital, two to nursing homes, and one to the patient's own home); four of these were ventilated through a tracheostomy and one was extubated and required ventilation through a facemask.

\section{NPD group of patients}

MND was diagnosed in the 17 patients in the NPD group during the course of their admission once it became clear that neuromuscular weakness was a major factor in the respiratory failure (table 2). Five patients in the NPD group died on ITU. All had major muscle wasting and weight loss in the months before admission and died after several attempts to wean them from the ventilator had failed. In three of these patients the tracheostomy had been uncuffed but, in two, it remained cuffed. Each patient eventually developed bronchopneumonia from presumed aspiration. After discussion with the patient and their carers antibiotics were not given to any of the patients. The terminal stages were managed in close liaison with palliative care colleagues in both centres.

Twelve patients $(71 \%)$ were discharged from ITU, 11 of whom required ventilatory support. Of these, four went home and eight remained in hospital. Eight patients received IPPV through an uncuffed tracheostomy, one through a cuffed tracheostomy, and one through a nasal mask. One patient was successfully extubated and went home requiring no ventilatory support and one patient required a rocking bed at night.

There was considerable variation in the time between intubation and death (mean 7.4 months, median 2.8 months, range 1 week to 3.5 years, $n=22$; one patient was still living at the end of the study and one patient was lost to follow up). The PD group survived longer once ventilated (median 11 months, $\mathrm{n}=7$ ) than the NPD group (median three months, $\mathrm{n}=17$ ). For the patients discharged from ITU, the median time taken to be stabilised and weaned before discharge was 10 weeks (range 2 weeks to 20 months).

\section{DISCUSSION}

There are difficult management issues to consider when a patient with MND presents with respiratory failure, and tracheal intubation and artificial ventilation have already been initiated acutely. This series of 24 patients shows that after intubation, long term ventilatory support is often required. Seventeen patients were intubated before MND was diagnosed but in the remaining seven patients the diagnosis had been established although the development of respiratory failure had not been predicted.

Respiratory failure should be anticipated in all patients once MND is diagnosed. Optimal management of patients with MND includes protecting autonomy, giving information in advance of deterioration (particularly with regard to respiratory failure), addressing all aspects of care in a multidisciplinary environment, and discussing regularly any advance directives ${ }^{17}$. After careful discussion many patients decide to use non-invasive ventilatory support if their respiratory function deteriorates and is symptomatic. Patients who require non-invasive respiratory support for increasing periods of time while awake, or even continuously, may choose to undergo tracheostomy ${ }^{14}$. There are significant regional and cultural differences in the provision and uptake of these options. The discussion regarding their wishes may not have been initiated early enough in the course of the disease, and in the emergency environment it is rarely appropriate or indeed possible to discuss such issues. Some patients develop a rapidly progressive form of the condition causing a sudden and unpredicted decline in respiratory function or they may develop bronchopneumonia without overt evidence of bulbar weakness. These patients may present with respiratory failure before detailed management discussions have been possible.

The decision to undertake "elective" tracheostomy for patients with MND is difficult. The use of an uncuffed tracheostomy has been advocated for patients with progressive respiratory muscle weakness before commencing ventilatory 
support; for those who require increasing ventilatory support that cannot be adequately provided with nasal IPPV; or for those who are unable to tolerate the distress and discomfort of wearing a mask for up to 24 hours each day. However, tracheostomy carries a significant risk of complications and considerable difficulties in domiciliary management. Furthermore, although pulmonary aspiration may still occur with an uncuffed tube, there remains a concern that tracheostomy may lead to prolonged survival in the face of severe disability.

In the present series all 24 patients were intubated, ventilated, and admitted to ITUs. In some patients who have been intubated with a tracheal tube, it may be considered inappropriate to proceed to tracheostomy because of the patient's wishes and severe coexisting disability. However, in the present series all patients underwent tracheostomy. Considerations pertaining to the decision to undertake a tracheostomy in a patient with MND already intubated with an endotracheal tube differ from those outlined above for elective ventilatory support. Tracheostomy may be undertaken after careful discussion with patients and their carers to alleviate distress, discomfort, and the high risk of complications due to prolonged tracheal intubation. Secondly, tracheostomy may ease ventilation and airway management. Thirdly, tracheostomy may facilitate, where possible, weaning from ventilatory support. Finally, an uncuffed tracheostomy may allow the patient to remain independent while receiving long term continuous domiciliary support using IPPV.

Once the patient has been established and stabilised on ventilatory support, with or without a tracheostomy, weaning from IPPV should follow a stepwise process. This involves extubation and non-invasive ventilatory support progressing ultimately to total independence if possible. However, a proportion of patients who have been stabilised on IPPV through tracheostomy but who have severe respiratory muscles weakness may fail to wean adequately. This may present severe difficulties in management, as failure to wean from a cuffed tracheostomy means the patient is unable to swallow or speak and is likely to develop progressive muscular weakness resulting in total paralysis of volitional movement. Despite the severity of weakness, the patient may be protected, by the cuff, from the development of aspiration and bronchopneumonia.

In the present series five patients receiving ventilatory support through an uncuffed tube and two of those supported through a cuffed tube died. In each case death was associated with the development of an overwhelming bronchopneumonia, which, after careful discussion, was left untreated by antibiotics in four patients. In no case was a decision made to reduce ventilatory support in a planned manner in the absence of underlying infection. However, the two patients who had a cuffed tracheostomy elsewhere that could not be uncuffed did survive for prolonged periods with severe disability. Both these patients were able to indicate that it was their wish to be maintained for as long as possible in such a state. One patient lived for 15 months with no limb movements or speech, although he was able to communicate his wishes by eye movements and blinks.

The terminal stages of the disease should be managed in close liaison with palliative care physicians. ${ }^{26}{ }^{27}$ Overwhelming bronchopneumonia results in failure of pulmonary gas exchange, generalised sepsis, and death even in patients receiving ventilatory support. The aim of terminal care is to anticipate the development of overwhelming sepsis and to relieve dyspnoea and anxiety while ventilatory support is reduced. All the patients were treated with morphine and anxiolytic medication during the terminal stages of their illness.

Several authors have discussed acute respiratory failure in patients with MND. De Carvalho et al ${ }^{22}$ described a series of 29 patients, of whom 18 presented acutely and 13 required some form of ventilatory support. Many of these patients presented with predominantly upper limb wasting and weakness, suggesting that some may have had progressive muscular atrophy or even multifocal motor neuropathy. It was not clear how many of these patients were known to have MND before the initiation of respiratory support. In the present series all the patients had probable or definite MND as defined by the El Escorial criteria and few had a predominantly upper limb emphasis. Chen et $\mathrm{l}^{23}$ described seven patients with MND who developed acute respiratory failure of uncertain aetiology. None of these patients was successfully weaned and six died soon after admission.

Although artificial ventilation may increase life expectancy ${ }^{9-14}$ there are many concerns about instituting such respiratory support, particularly in the absence of detailed discussion with the patient and family. These centre on the questionable quality of life on respiratory support and disease progression leading to a totally "locked in" state. ${ }^{11}$ Offering ventilatory support electively allows patients and families to discuss these issues, come to terms with the disease, and increase familiarity with the equipment, possibly increasing compliance. ${ }^{13}{ }^{15}$ Families should not have to make decisions, if avoidable, during an acute life threatening deterioration. Ideally there should be discussions about respiratory support with the patient and family soon after MND has been diagnosed. ${ }^{28}{ }^{29}$ Not all patients wish to be ventilated, partly because of the fear of becoming profoundly paralysed and totally dependent. Close surveillance of patients with MND increases the chance of the onset of respiratory failure being detected early but, as this series shows, symptoms alone are not an indicator of incipient respiratory failure. ${ }^{30-36}$ Patients with MND presenting in respiratory failure without an established diagnosis will continue to be a challenge.

The results of our study show that when a patient with MND is ventilated acutely, with or without a diagnosis, independence from the ventilator is rarely achieved. Almost all of the patients need long term ventilatory support and the degree of respiratory support increases with time as the underlying disease progresses. The aim of management of these patients should be weaning the patient onto the minimum support compatible with symptomatic relief and comfort.

\section{Authors' affiliations}

M D Bradley, *R W Orrell, Department of Clinical Neurosciences, Royal Free and University College Medical School, Royal Free Campus, London NW3 2QG, UK

J Clarke, D M Kullmann, N Hirsch, **R S Howard, Batten-Harris Unit, National Hospital for Neurology and Neurosurgery, Queen Square, London WCIN 3BG, UK

A C Davidson, A J Williams, Lane Fox Unit, St Thomas' Hospital, London SE 1 7EH, UK

*Also the Batten-Harris Unit, National Hospital for Neurology and Neurosurgery

* *Also the Lane Fox Unit, St Thomas' Hospital

Competing interests: none declared

\section{REFERENCES}

1 Ringel SP, Murphy JR, Alderson MK, et al. The natural history of amyotrophic lateral sclerosis. Neurology 1993;43:1316-22.

2 Haverkamp LJ, Appel V, Appel SH. Natural history of amyotrophic lateral sclerosis in a database population. Validation of a scoring system and a model for survival prediction. Brain 1995;1 18:707-19.

3 Polkey MI, Lyall RA, Green M, et al. Expiratory muscle function in amyotrophic lateral sclerosis. Am J Crit Care Med 1998;158:734-41. 4 Hardiman $\mathrm{O}$. Symptomatic treatment of respiratory and nutritional failure in amyotrophic lateral sclerosis. J Neurol 2000;247:245-51.

5 Howard RS, Wiles CM, Loh L. Respiratory complications and their management in motor neuron disease. Brain 1989;112:1155-70.

6 Evangelista T, de Carvalho M, Pinto A, et al. Phrenic nerve conduction in amyotrophic lateral sclerosis. J Neurol Sci 1995;129(suppl):35-7.

7 Lyall RA, Donaldson N, Polkey MI, et al. Respiratory muscle strength and ventilatory failure in amyotrophic lateral sclerosis. Brain $2001 ; 124: 2000-13$. 
8 Hadijoutis S, Eccles R, Wiles CM. Coughing and choking in motor neurone disease. J Neurol Neurosurg Psychiatry 2000;68:601-4.

9 Hayashi H, Kato S, Kawada A. Amyotrophic lateral sclerosis patients living beyond respiratory failure. J Neurol Sci 1991;105:73-8.

10 Hayashi $\mathbf{H}$. Ventilatory support: Japanese experience. J Neurol Sci 1997;152(suppl 1):97-100.

11 Hayashi H, Kato S. Total manifestations of amyotrophic lateral sclerosis. ALS in the totally locked-in state. J Neurol Sci 1989;93:19-35.

12 Hill NS. Noninvasive ventilation positive pressure ventilation in neuromuscular disease. Enough is enough. Chest 1995;105:337-8.

13 Aboussouan LS, Khan SU, Meeker DP, et al. Effect of non-invasive positive pressure ventilation on survival in amyotrophic lateral sclerosis. Ann Intern Med 1997; 127:450-3.

14 Cazzolli PA, Oppenheimer EA. Home mechanical ventilation for amyotrophic lateral sclerosis: nasal compared to tracheostomy-intermittent positive pressure ventilation. J Neurol Sci 1996;139(suppl):S123-8.

15 Melo J, Homma A, Iturriaga $\mathrm{E}$, et al. Pulmonary evaluation and prevalence of non-invasive ventilation in patients with amyotrophic lateral sclerosis: a multicenter survey and proposal of a pulmonary protocol. J Neurol Sci 1999:169:114-7.

16 Gelinas DF, O'Connor P, Miller RG. Quality of life for ventilator dependent ALS patients and their caregivers. J Neurol Sci 1998;160(suppl 1):S134-6

17 Miller RG, Rosenberg JA, Gelinas DF, et al. Practice parameter: the care of the patient with amyotrophic lateral sclerosis (an evidence based review). Neurology 1999;52:1311-23.

18 Hillberg RE, Johnson DC. Non-invasive ventilation. N Engl J Med 1997;337: 1746-52

19 Pinto AC, Evangelista T, Carvalho M, et al. Respiratory assistance with a non-invasive ventilator (Bipap) in MND/ALS patients: survival rates in a controlled trial. J Neurol Sci 1995;129:S19-26.

20 Kleopa AK, Sherman M, Neal B, et al. Bipap improves survival and rate of pulmonary function decline in patients with ALS. J Neurol Sci 1999;164:82-8.

21 Carrey Z, Gottfried SB, Levy RD. Ventilatory muscle support in respiratory failure with nasal positive pressure ventilation. Chest 1990;97:150-8
22 De Carvalho M, Matias T, Coelho F, et al. Motor neurone disease presenting with respiratory failure. J Neurol Sci 1996;139(suppl): 117 22.

23 Chen R, Grand'Maison F, Strong M, et al. Motor neuron disease presenting as acute respiratory failure: a clinical and pathological study. J Neurol Neurosurg Psychiatry 1996;60:455-8.

24 Annane D, Korach JM, Templier F, et al. Diaphragmatic paralysis preceding amyotrophic lateral sclerosis. Lancet 1993;342:990-1.

25 El Escorial. World Federation of Neurology criteria for the diagnosis of amyotrophic lateral sclerosis. J Neurol Sci 1994;124(suppl):97-107.

26 Borasio GD, Volz R, Miller RG. Palliative care in amyotrophic lateral sclerosis. Neurol Clin 2001;19:829-48.

27 Gelinas DF, Miller RG. A treatable disease: a guide to the management of amyotrophic lateral sclerosis. In: Brown RH, Meninger V, Swash M, eds. Amyotrophic lateral sclerosis. London: Martin Dunitz 2000:405-22.

28 Albert SM, Murphy PL, Del Bene ML, et al. Prospective study of palliative care in ALS: choice, timing and outcomes. J Neurol Sci 1999: 169: 108-13.

29 Albert SM, Murphy PL, Del Bene ML, et al. A study of preferences and actual treatment choices in ALS. Neurology 1999;53:278-83.

30 Hopkins LC, Tatarian GT, Pianta TF. Management of ALS: respiratory care. Neurology 1996;47(suppl 2):123-5.

31 Fallat RJ, Jewitt B, Bass $M$, et al. Spirometry in amyotrophic lateral sclerosis. Arch Neurol 1979;36:74-80.

32 Brooks BR. Natural history of ALS: symptoms, strength, pulmonary function, and disability. Neurology 1996;47:S71-82.

33 Schiffman PL, Belsh JM. Pulmonary function at diagnosis of amyotrophic lateral sclerosis. Rate of deterioration. Chest 1993;103:508-13.

34 Stambler N, Charatan M, Cederbaum JM, ALS CNTF Treatment Study Group. Prognostic indicators of survival in ALS. Neurology 1998:50:66-72.

35 David WS, Bundlie SR, Mahdavi Z. Polysomnographic studies in amyotrophic lateral sclerosis. J Neurol Sci 1997;152(suppl):S29-35.

36 Kimura K, Tachibana N, Kimura J, et al. Sleep-disordered breathing at an early stage of amyotrophic lateral sclerosis. J Neurol Sci $1999 ; 164: 37-43$

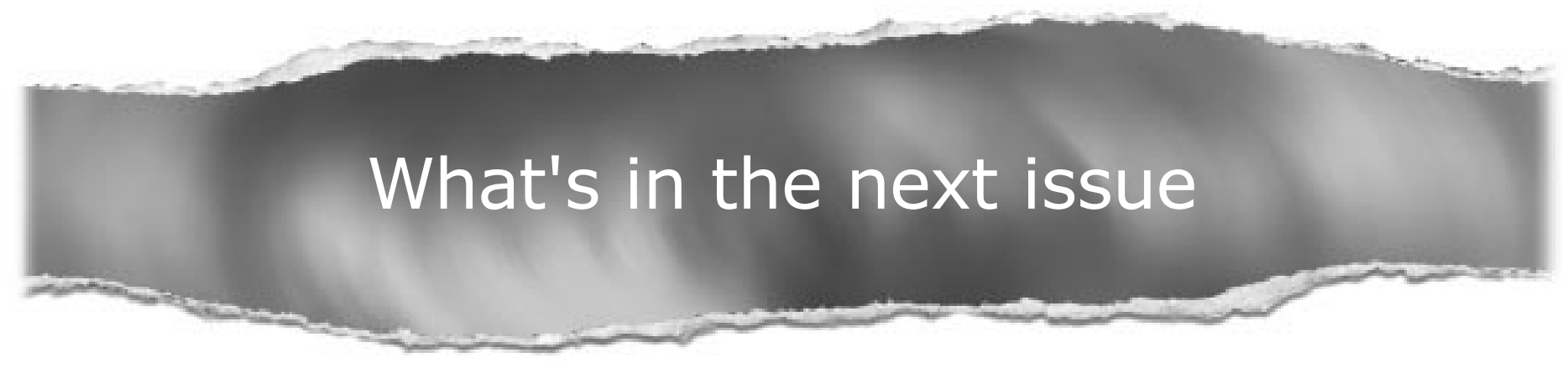

Future content

See which articles have just been accepted for publication and preview the table of contents for the next issue a month before it is published

www.jnnp.com 